Mh. Math. 100, 113-120 (1985)

\title{
Classical Families of Univalent Functions in the Hornich Space
}

\author{
By \\ Wolfram Koepf, Berlin \\ (Received 29 October 1984; in revised form 22 May 1985)
}

\begin{abstract}
In this paper the simple structure between some convex sets in the Banach space $H$ introduced by Hornich is used to determine the extreme points of the families $K(\alpha)$ of convex functions of order $\alpha$ and $V(k)$ of functions with bounded boundary rotation $k \pi$. For close-to-convex functions of order $\beta, \beta \in] 0,1[$, a partial result is given. The results for $K(\alpha)$ and $V(k)$ agree with those that hold for the closed convex hulls of the same families with respect to the usual linear structure and the topology of locally uniform convergence. However, in this case, for $k \in] 2,4[$ the question of determining the extreme points of $\overline{c o} V(k)$ is still open.
\end{abstract}

1. Introduction. Let $A$ be the set of all analytic functions in the unit disk $\mathbb{D}$. Further let $H$ denote the subclass of all locally univalent functions $f$ which are normalized by the conditions

$$
f(0)=0, f^{\prime}(0)=1
$$

and for which $\arg \left(f^{\prime}\right)$ is bounded in $\mathbb{D}$. Here arg denotes that branch of the imaginary part of the logarithm which vanishes at the point 1 .

HoRNICH [5] showed that $\left(H, \oplus, \odot,\|\|_{H}\right)$ is a Banach space with the operations

$$
\begin{gathered}
(f \oplus g)(z):=\int_{0}^{z} f^{\prime}(\zeta) \cdot g^{\prime}(\zeta) d \zeta \\
(\lambda \odot f)(z):=\int_{0}^{z}\left(f^{\prime}(\zeta)\right)^{\lambda} d \zeta \text { and } \\
\|f\|_{H}:=\sup _{z_{1} \in \mathbb{D}} \sup _{z_{2} \in \mathbb{D}}\left(\arg f^{\prime}\left(z_{2}\right)-\arg f^{\prime}\left(z_{1}\right)\right) .
\end{gathered}
$$

The null element in $H$ is the identity mapping. Sequential convergence with respect to the norm \|\|$_{H}$ implies locally uniform convergence but not conversely (see [5], p. 39).

We begin with the discussion of some properties of families of analytic functions considered as subsets of $H$. A univalent function is 
called convex if it has a convex range. Let $K$ denote the family of convex functions, that are normalized by (1). It is well-known, that a locally univalent normalized function $f$ is in $K$ if and only if

where

$$
1+\frac{z f^{\prime \prime}}{f^{\prime}} \in P
$$

$$
P:=\{f \in A \mid \operatorname{Re} f>0, f(0)=1\} .
$$

Because of the Herglotz integral representation for functions in $P$ (see e.g. [11], p. 4) an equivalent condition for $f \in K$ is that

$$
\log f^{\prime}(z)=\int_{X} \log \frac{1}{(1-x z)^{2}} d \mu
$$

where $\mu$ is a Borel probability measure on $X:=\{x \in \mathbb{C}|| x \mid=1\}$. From (3) it is easily seen that

$$
\begin{aligned}
\arg f^{\prime}(z) & =\int_{X} \arg \frac{1}{(1-x z)^{2}} d \mu=-2 \int_{X} \arg (1-x z) d \mu \leqslant \\
& \leqslant 2 \arcsin |z|
\end{aligned}
$$

with equality if and only if $\mu$ is a point measure, i. e. $f$ has the form

$$
f(z)=\frac{z}{1-x z} \text { for some } x \in X .
$$

The last assertion follows from the fact that the integral is the limit of finite convex combinations of points that lie in the closed disk with radius $|z|$ and centre at the point 1 . The bound given in (4), which is due to MARX [7], Theorem $\mathbf{B}$, shows that $K$ is a subset of the ball in $H$ with radius $2 \pi$ (änd centre at the null element).

We note that as another easy and well-known consequence of (3),

$$
\left|f^{\prime \prime}(0)\right|=\left|\frac{f^{\prime \prime}}{f^{\prime}}(0)\right|=2\left|\int_{X} x d \mu\right| \leqslant 2
$$

with equality if and only if $\mu$ is a point measure, i. e., $f$ has the form (5).

From (2) it is easily seen that $K$ is closed and convex (see [3], Theorem 6.1). Furthermore, since $K$ is the continuous image of the compact set of Borel probability measures on $X$ with respect to the weak topology, $K$ is compact. The family of convex functions of order $\alpha, \alpha<1$, normalized by (1), will be denoted by $K(\alpha)$. These classes had 
been introduced (in a slightly modified form) by ROBERTSON [10], p. 383. A locally univalent normalized function $f$ is in $K(\alpha)$ if and only if

$$
1+\frac{z f^{\prime \prime}}{(1-\alpha) f^{\prime}} \in P .
$$

As above it is easy to show that $K(\alpha)$ is a subset of the ball in $H$ with radius $2(1-\alpha) \pi$. Let $V(k)$ denote the families of functions with bounded boundary rotation $k \pi$ for some $k \geqslant 2$, normalized by (1), which had been studied by PAATERo [8]. A locally univalent normalized function is in $V(k)$ if and only if

$$
1+\frac{z f^{\prime \prime}}{f^{\prime}}=\left(\frac{k}{4}+\frac{1}{2}\right) p_{1}-\left(\frac{k}{4}-\frac{1}{2}\right) p_{2}
$$

for some $p_{1}, p_{2} \in P$ (see [2], p. 344). Let $C(\beta)$ denote the family of closeto-convex functions of order $\beta$ for some $\beta \geqslant 0$, normalized by (1), which had been introduced by KAPLAN [6] and POMMERENKE [9]. With the notation

$$
P_{0}:=\left\{f \in A \mid \exists \delta \in \mathbb{R}: \operatorname{Re}\left(e^{i \delta} f\right)>0, f(0)=1\right\},
$$

a locally univalent normalized function is in $C(\beta)$ if and only if there is a function $\varphi \in K$ such that $f^{\prime} / \varphi^{\prime}=p^{\beta}$ for some $p \in P_{0}$.

From the definition we see that $f \in C(\beta)$ implies

$$
\begin{gathered}
\arg f^{\prime}\left(z_{2}\right)-\arg f^{\prime}\left(z_{1}\right)= \\
=\left(\arg \varphi^{\prime}\left(z_{2}\right)-\arg \varphi^{\prime}\left(z_{1}\right)\right)+\beta\left(\arg p\left(z_{2}\right)-\arg p\left(z_{1}\right)\right)< \\
<2 \pi+\beta \pi=(2+\beta) \pi
\end{gathered}
$$

because of (4) and because $p \in P_{0}$. Thus $C(\beta)$ is a subset of the ball in $H$ with radius $(2+\beta) \pi$. All families considered are closed and convex. We want to recall now some inclusion relations between the classes considered. An easy consequence of the representation (7) is

Lemma 1. For every $\alpha<0$ we have $K(\alpha) \subset V(2-4 \alpha)$.

The following Lemma is well-known.

Lemma 2 (BRANNAN [2], Theorem 2.2). For every $k \geqslant 2$ we have $V(k) \subset C(k / 2-1)$.

Thus $V(k)$ is a subset of the ball in $H$ with radius $(1+k / 2) \pi$. If $V$ is a vector space and $F \subset V$, then $f$ is called an extreme point of 
$F$ if there is no proper convex representation

$$
\left.f=t f_{1}+(1-t) f_{2}, \quad t \in\right] 0,1[,
$$

with suitable functions $f_{1}, f_{2} \in F, f_{1} \neq f_{2}$.

Recall that the extreme points of the convex set $P$ with respect to the usual linear structure are exactly the functions $f$ of the form

$$
f(z)=\frac{1+x z}{1-x z}, \quad x \in X
$$

(see e.g. [11], Theorem 1.5).

This statement is equivalent to the Herglotz respresentation and implies that the extreme points of the convex set $K$ with respect to the Hornich space structure are exactly the functions of the form (5), which is a result due to Cima and Pfaltzgraff [3], Theorem 6.2.

This result will be important in the next section and we refer to it by $C P$.

2. Families of univalent functions in the Hornich space. The following Lemma shows that $K(\alpha)$ is a linear and continuous image of $K$ with respect to the Hornich space structure.

Lemma 3 (see e. g. [1], p. 5). For all $\alpha<1$ we have

$$
K(\alpha)=(1-\alpha) \odot K \text {. }
$$

An immediate consequence is

Corollary 4. For all $\alpha<1$ the family $K(\alpha)$ is a compact subset of $H$. $A$ function $f$ is an extreme point of $K(\alpha)$ with respect to the Hornich space structure if and only if

$$
f^{\prime}(z)=\frac{1}{(1-x z)^{2(1-\alpha)}} \text { for some } x \in X .
$$

Proof. For each $\alpha<1$ the mapping

$$
L: H \rightarrow H, f \mapsto(1-\alpha) \bigodot f
$$

is a homeomorphism and Lemma 3 shows that $L(K)=K(\alpha)$. This and $C P$ imply the Corollary.

At this stage we insert the following elementary Lemma.

Lemma 5. Let $F$ and $G$ be subsets of the linear space $V$. Then an extreme point of the set $F \oplus G$ is the sum of extreme points of $F$ and $G$ respectively. 
Proof. It is easily seen that a proper convex representation for some $f \in F$ within the class $F$ defines a proper convex representation for $f \oplus g$ within $F \oplus G$ for arbitrary $g \in G$. A similar argument interchanging $f$ and $g$ implies the result.

Now we get the following important

Theorem 6. For every $\alpha, \beta \in \mathbb{R}^{+}$the family $(\alpha \odot K \ominus \beta \odot K$ ) is a compact subset of $H$ whose extreme points are exactly the functions $f$ of the form

$$
f^{\prime}(z)=\frac{(1+x z)^{2 \beta}}{(1-y z)^{2 \alpha}}
$$

with some $(x, y) \in X^{2}, x \neq-y$.

Proof. ( $\alpha \odot K \ominus \beta \odot K$ ) is the continuous image of $K$ and so compact. From Lemma 5 and $C P$ it follows that every extreme point of $(\alpha \odot K \ominus \beta \odot K)$ has the form (8) for some $x, y \in X$.

If $x=-y$, then the function defined by (8) lies on the straight line connecting the identity mapping with the function $\operatorname{sign}(\alpha-\beta)$. $\cdot \max (\alpha, \beta) \odot \frac{z}{1-y z}$, which are both functions of $(\alpha \odot K \ominus \beta \odot K)$, and thus cannot be extreme.

So we have shown that an extreme point has the form (8) for some $(x, y) \in X^{2}, x \neq-y$.

It remains to show that every point of this form is extreme in $(\alpha \odot K \ominus \beta \odot K)$. Therefore we shall show that each of these functions $f$ maximizes a functional uniquely over $(\alpha \odot K \ominus \beta \odot K)$ that is linear and continuous with respect to the Hornich space structure. This shows that all considered functions are extreme.

For $z_{0} \in \mathbb{D} \backslash\{0\}$ the functionals $\pm \arg f^{\prime}\left(z_{0}\right)$ are linear and continuous in the Hornich space.

For each $f \in(\alpha \odot K \ominus \beta \odot K)$ we get

$$
\arg f^{\prime}\left(z_{0}\right)=\alpha \cdot \arg g^{\prime}\left(z_{0}\right)-\beta \cdot \arg h^{\prime}\left(z_{0}\right)
$$

with some $g, h \in K$, and thus it follows from (4)

$$
\left|\arg f^{\prime}\left(z_{0}\right)\right| \leqslant(\alpha+\beta) \cdot 2 \arcsin \left|z_{0}\right|
$$

with equality if and only if

$$
g(z)=\frac{z}{1-y z} \text { and } h(z)=\frac{z}{1+x z}
$$


for some $(x, y) \in X^{2}$, i. e.

$$
f_{x y}^{\prime}(z)=\frac{(1+x z)^{2 \beta}}{(1-y z)^{2 \alpha}} .
$$

Equality in (9) occurs if and only if

$$
\arg \left(-x z_{0}\right)=-\arccos \left|z_{0}\right|, \arg \left(y z_{0}\right)=\arccos \left|z_{0}\right|
$$

or

$$
\arg \left(x z_{0}\right)=\arccos \left|z_{0}\right|, \arg \left(y z_{0}\right)=-\arccos \left|z_{0}\right| .
$$

If (10) holds, then $\arg f_{x y}^{\prime}\left(z_{0}\right)=(\alpha+\beta) \arcsin \left|z_{0}\right|$, and thus the functional $\arg f^{\prime}\left(z_{0}\right)$ is maximized, and if (11) holds, then $\arg f_{x y}^{\prime}\left(z_{0}\right)=-(\alpha+\beta) \arcsin \left|z_{0}\right|$, and thus the functional $-\arg f^{\prime}\left(z_{0}\right)$ is maximized.

Now we have only to observe that for every $z_{0} \in \mathbb{D} \backslash\{0\}$ and each of (10) and (11) there is exactly one solution $(x, y) \in X^{2}$ with $x^{2} \neq y^{2}$. Conversely for each pair $(x, y) \in X^{2}, x^{2} \neq y^{2}$, there is a (unique) point $z_{0} \in \mathbb{D} \backslash\{0\}$, such that one of (10) or (11) holds.

Thus it follows that if $x^{2} \neq y^{2}$, then the functions considered maximize one of the functionals $\pm \arg f^{\prime}\left(z_{0}\right)$ uniquely and thus are extreme points.

If $x^{2}=y^{2}$, then either $x=-y$, and in this case we had shown already that $f_{x y}$ is not extreme, or $x=y$, and then the functions $f_{x y}$ maximize uniquely a functional of the type $\operatorname{Re} w f^{\prime \prime}(0), w \in \mathbb{C}$.

To prove this, we observe that for $f \in(\alpha \odot K \ominus \beta \odot K)$

$$
\frac{f^{\prime \prime}}{f^{\prime}}(0)=\alpha \frac{g^{\prime \prime}}{g^{\prime}}(0)-\beta \frac{h^{\prime \prime}}{h^{\prime}}(0)
$$

with some $g, h \in K$. From (6) we see that

$$
\left|\frac{f^{\prime \prime}}{f^{\prime}}(0)\right| \leqslant 2(\alpha+\beta)
$$

with equality if and only if

$$
g(z)=\frac{1}{1-x z} \text { and } h(z)=\frac{z}{1+x z}
$$

for some $x \in X$, i.e.

$$
f^{\prime}(z)=\frac{(1+x z)^{2 \beta}}{(1-x z)^{2 \alpha}} .
$$


Because these functionals are continuous and linear in $H$, it follows that for all $x \in X$ the function $f_{x x}$ is an extreme point, too.

The family $V(k)$ is of the same type.

Lemma 7 (BRANNAN [2], Theorem 3.1). For all $k>2$ we have

$$
V(k)=\left(\frac{\mathrm{k}}{4}+\frac{1}{2}\right) \odot K \ominus\left(\frac{\mathrm{k}}{4}-\frac{1}{2}\right) \odot K .
$$

Corollary 8. For all $k>2$ the family $V(k)$ is a compact subset of $H$. $A$ function $f$ is an extreme point of $V(k)$ with respect to the Hornich space structure if and only if

$$
f^{\prime}(z)=\frac{(1+x z)^{k / 2-1}}{(1-y z)^{k / 2+1}} \text { for some }(x, y) \in X^{2}, x \neq-y .
$$

Proof. Lemma 7 and Theorem 6 give the result.

TRIMBle and Wright [12], Theorem 3, determined the set of extreme points in $C:=C(1)$, the set of close-to-convex functions. This set includes the extreme points of $V(4)$ (the latter is a proper subfamily of $C$, as Lemma 2 shows), but is much bigger.

We shall give a characterization of the extreme points of $C(\beta)$ for $\beta \in] 0,1$ [ in terms of the extreme points of $K$ and $C$. This will follow from

Lemma 9. For all $\beta \in[0,1]$ we have

$$
C(\beta)=(1-\beta) \odot K \oplus \beta \odot C .
$$

Proof. " $\subset$ ": Let be $f \in C(\beta)$ with suitable $\varphi \in K$ and $p \in P_{0}$ such that $f^{\prime}=\varphi^{\prime} \cdot p^{\beta}$. The function $g$ defined by $g^{\prime}=\varphi^{\prime} \cdot p$ lies in $C$ and it follows

$$
f^{\prime}=\left(\varphi^{\prime}\right)^{1-\beta} \cdot\left(g^{\prime}\right)^{\beta},
$$

which proves that $f \in(1-\beta) \odot K \oplus \beta \odot C$.

"د": For $\varphi \in K$ and $g \in C$ with suitable $\psi \in K, p \in P_{0}$, satisfying $g^{\prime}=\psi^{\prime} \cdot p$, we define $f$ by (12). Then the function $\Phi$ with $\Phi^{\prime}=\left(\varphi^{\prime}\right)^{1-\beta} \cdot\left(\psi^{\prime}\right)^{\beta}$ is convex and we get

$$
\frac{f^{\prime}}{\Phi^{\prime}}=\frac{\left(\varphi^{\prime}\right)^{1-\beta} \cdot\left(g^{\prime}\right)^{\beta}}{\left(\varphi^{\prime}\right)^{1-\beta} \cdot\left(\psi^{\prime}\right)^{\beta}}=\left(\frac{g^{\prime}}{\psi^{\prime}}\right)^{\beta}=p^{\beta},
$$

and thus $f \in C(\beta)$. 
Corollary 10. If $\beta \in] 0,1[$ then an extreme point $f$ of $C(\beta)$ with respect to the Hornich space structure satisfies

$$
f^{\prime}(z)=\frac{\left(g^{\prime}\right)^{\beta}}{(1-x z)^{2(1-\beta)}}
$$

for some $x \in X$ and some extreme point $g$ of $C$.

Proof. Lemmata 5 und 9 give this result.

\section{References}

[1] Aharonov, D., Friedland, S.: On functions of bounded boundary rotation. Ann. Acad. Sci. Fenn. Ser. A I. Math. 585, 1-18 (1974).

[2] Brannan, D. A.: On functions of bounded boundary rotation I. Proc. Edinburgh Math. Soc. 16, 339-347 (1968/69).

[3] Cima, J.A., Pfaltzgraff, J.A.: A Banach space of locally univalent functions. Mich. Math. J. 17, 321-334 (1970).

[4] Goodman, A. W.: Univalent Functions. Vol. II. Tampa, Florida: Mariner Publ. Co. 1983.

[5] HoRNICH, H.: Ein Banachraum analytischer Funktionen in Zusammenhang mit den schlichten Funktionen. Mh. Math. 73, 36-45 (1969). (1952).

[6] KAPLAN, W.: Close-to-convex schlicht functions. Mich. Math. J. 1, 169-185

[7] MARX, A.: Untersuchungen über schlichte Abbildungen. Math. Ann. 107, $40-67(1932)$.

[8] PAATERo, V.: Über die konforme Abbildung von Gebieten, deren Ränder von beschränkter Drehung sind. Ann. Acad. Sci. Fenn. Ser. A. 33:9, 1-78 (1931).

[9] Pommerenke, Ch.: On close-to-convex analytic functions. Trans. Amer. Math. Soc. 114, 176-186 (1965).

[10] RoBERTSON, M. S.: On the theory of univalent functions. Ann. Math. 37, $374-408$ (1936).

[11] Schober, G.: Univalent Functions - Selected Topics. Lect. Notes Math. 478. Berlin-Heidelberg-New York: Springer. 1975.

[12] TRIMBLE, S. Y., WRIGHT, D. J.: Close-to-convex functions and their extreme points in Hornich space. Mh. Math. 85, 235-244 (1978).

WOLFRAM KoEPF

Freie Universität Berlin

Fachbereich Mathematik

I. Mathematisches Institut

Arnimallee 3

D-1000 Berlin 33 